\title{
Nuclear Shapes from Alpha-Gamma-Ray Angular Correlations
}

\author{
Z. Majka, (a) D. G. Sarantites, L. G. Sobotka, K. Honkanen, ${ }^{(b)}$ E. L. Dines, ${ }^{(c)}$ L. A. Adler, and Li Ze \\ Department of Chemistry, Washington University, St. Louis, Missouri 63130
}

M. L. Halbert, J. R. Beene, and D. C. Hensley

Oak Ridge National Laboratory, Oak Ridge, Tennessee 37830

and

R. P. Schmitt and G. Nebbia

Cyclotron Institute and Department of Chemistry, Texas A\&M University, College Station, Texas 77840

(Received 27 October 1986)

\begin{abstract}
The angular distribution of alpha particles with respect to the spin direction of the residual nuclei is used to probe the shapes of the emitting systems. Two compound systems have been studied: ${ }^{170} \mathrm{Yb}$ and ${ }^{110} \mathrm{Sn}$. Deformed emission shapes are required in order to understand the alpha-gamma correlations in the ${ }^{170} \mathrm{Yb}$ system, but not for the ${ }^{110} \mathrm{Sn}$ compound system. These conclusions are identical to those reached by the study of giant resonances built on excited states for very similar systems.
\end{abstract}

PACS numbers: $25.70 . \mathrm{Gh}, 23.20 . \mathrm{En}$

The desire to explore the large-scale collective motion in small quantal systems has been the stimulus for the study of nuclear shapes. With heavy-ion reactions these studies can be extended to highly excited and rapidly rotating nuclei. Recently, much attention has been focused on the decay of giant resonances built on excited states as a probe of nuclear shapes in the high excitation energy and angular momentum regions. ${ }^{1,2}$ In this Letter, we present an alternative method for probing nuclear deformation, which we show is consistent with recent giantresonance work.

It has been known for some time that measurements of $\alpha$-particle emission from highly excited nuclei are sensitive to nuclear shapes. Conventionally, studies that use this probe examine angular distributions with respect to the beam direction. Since the correlation of interest is between the emission direction and that of the total angular momentum, one has to integrate over the possible spin directions and over all the angular momenta populated in each reaction system. However, by use of a $4 \pi$ $\gamma$-ray device such as the spin spectrometer ${ }^{3}$ it is possible to deduce the spin direction of the residual nuclei. ${ }^{4}$ Since the total angular momentum and the residual-spin direction are highly correlated in high-spin systems, one can dispense with the integration and simply study the $\alpha$-particle emission relative to the residual-spin direction.

In previous work, we have employed this technique and demonstrated that in the case of rare-earth compound systems the $\alpha-\gamma$ angular correlations suggest the influence of deformation. In the present work we show that very different characteristics are displayed by the $\alpha-\gamma$ correlations for the decay of a ${ }^{110} \mathrm{Sn}$ compound system, and that these characteristics are consistent with emission for a spherical nucleus. The same conclusion has been reached in giant-dipole-resonance (GDR) stud- ies of very similar compound systems. ${ }^{5}$ These results confirm the conjectures made in our earlier work ${ }^{4}$ and offer an illustration of the potential of these techniques.

The experiments were done at the Oak Ridge heavyion facility (HHIRF). The rare-earth compound systems was formed in the reaction $177-\mathrm{MeV}{ }^{20} \mathrm{Ne}+{ }^{150} \mathrm{Nd}$ as described in Ref. 4 . The ${ }^{110} \mathrm{Sn}$ compound system was produced at an excitation energy of $92.7 \mathrm{MeV}$ in bombardments of $320-450-\mu \mathrm{g} / \mathrm{cm}^{2}{ }^{65} \mathrm{Cu}$ self-supporting target foils, enriched to $99.7 \%$ in the mass-65 isotope, with a $200-\mathrm{MeV}{ }^{45} \mathrm{Sc}$ beam from the Holifield Heavy-Ion Research Facility. The $\alpha$ particles were detected by two large-solid-angle telescopes having ion chambers as $\Delta E$ and $\mathrm{Si}(\mathrm{Li})$ as $E$ detectors. Each $\mathrm{Si}(\mathrm{Li})$ detector consisted of four strips $(1.1 \mathrm{~cm}$ by $4.6 \mathrm{~cm})$ which were position sensitive in the longer dimension. Each telescope was centered at $55^{\circ}$ to the beam direction and subtended $18^{\circ}$ in the laboratory system.

The spin spectrometer served as the $\gamma$-ray detector and measured simultaneously the $\gamma$-ray multiplicity $M_{\gamma}$, the total $\gamma$-ray deexcitation energy $E^{*}$, and the $\gamma$-ray angular correlations. In this experiment 70 of the $72 \mathrm{NaI}$ detectors were used, covering $94.5 \%$ of $4 \pi$ sr. For each event triggered by either ion chamber the $\mathrm{NaI}$ pulse heights and times as well as the $\Delta E$ and $E$ pulse heights, the position, the $e^{-}$drift time, and the time for each $\mathrm{Si}(\mathrm{Li})$ strip were recorded on magnetic tape. The data were preprocessed to distinguish between $\gamma$-ray and neutron pulses by their associated times of flight. ${ }^{3}$

The distribution of the $\gamma$-ray coincidence fold $\left(k_{\gamma}\right)$ for events triggered by $\alpha$ particles detected at $90^{\circ}$ in the center of mass is shown in Fig. 1(a). It is seen that the $k_{\gamma}$ distribution, which is closely related to $M_{\gamma}$, indicates that the spin distribution extends to high angular momentum for this reaction system. The small bump at 

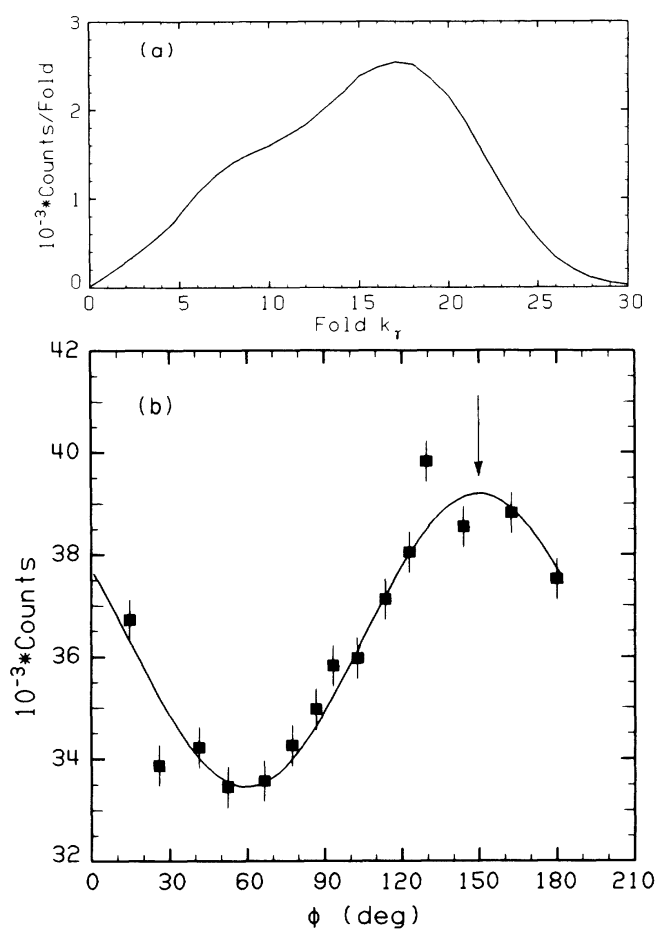

FIG. 1. (a) Distribution of the $\gamma$-ray coincidence fold $k_{\gamma}$ for events triggered by $\alpha$ particles detected near $\theta_{\text {c.m. }}=90^{\circ}$ from $200-\mathrm{MeV}{ }^{45} \mathrm{Sc}$ on ${ }^{65} \mathrm{Cu}$. (b) Angular distribution of $\gamma$-rays emitted in the plane perpendicular to the beam for events triggered by $\alpha$ particles observed at $\phi=150^{\circ}$ as indicated by the vertical arrow. The pattern is consistent with preponderance of stretched $E 2$ transitions. The error bars give estimates of the systematic errors; the statistical uncertanties are smaller by a factor of $\sim 2$. The solid curve is a least-squares fit to $A_{0}[1$ $\left.+A_{2} P_{2}(\cos \phi)\right]$.

$k_{\gamma} \sim 8$ is due to sequential emission from fissionlike decay channels. ${ }^{6}$ These channels transfer a substantial fraction of the available angular momentum into an orbital component and therefore are associated with low $M_{\gamma}$ or $k_{\gamma}$.

The factors that determine the spin orientation event by event from spin-spectrometer data have been outlined in detail in our earlier work. ${ }^{4}$ Treated quantitatively these considerations lead to spin-alignment response functions for the spectrometer. The only factor which varies significantly as a function of the nuclear system studied is the distribution in multipolarity of the $\gamma$ rays emitted in the reaction. The technique depends on the emission of a preponderance of $\gamma$ radiation with a particular angular relationship to the spin direction, for example, a preponderance of stretched quadrupole and/or nonstretched dipole $(\Delta I=0)$ over stretched dipole transitions. Fortunately, this can be checked by examination of the $\alpha-\gamma$ angular correlations. Figure 1(b) shows the distribution of $\gamma$ rays in the plane perpendicular to the

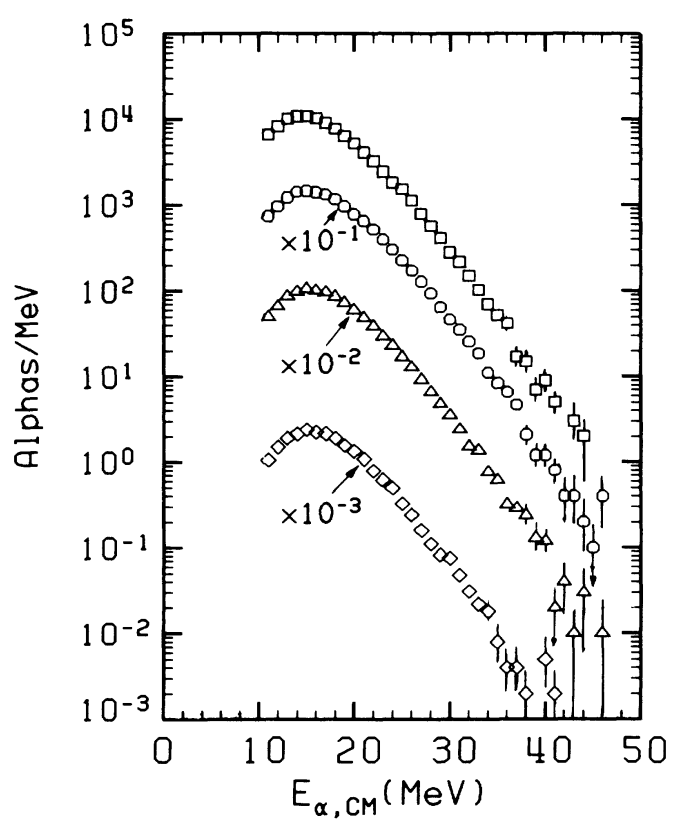

FIG. 2. $\alpha$-particle energy spectra observed at $\theta_{\text {c.m. }}=90^{\circ}$ corresponding to the following $k_{\gamma}$ bins: The squares, circles, triangles, and diamonds are for $k_{r}=9-13,14-18,19-23$, and $24-30$, respectively.

beam direction. Here the $\alpha$ particles were detected at an azimuthal angle $\phi=150^{\circ}$ and $k_{\gamma}$ was between 14 and 23 . This pattern is indeed consistent with a preponderance of stretched $E 2$ and/or nonstretched $(\Delta I=0)$ dipole transitions and justifies the use of the spin-alignment technique for obtaining the $\alpha$ angular distributions relative to the spin direction. Taking advantage of symmetry, we have shifted the points with $\phi>180^{\circ}$ downward by $180^{\circ}$ in Fig. 1(b) to make the distribution pattern more apparent.

The method employed for locating the spin direction becomes much more efficient and reliable, with only a slight loss in generality, if we assume that the spin direction of the residual nucleus after particle emission lies in the plane perpendicular to the beam direction (i.e., has not been significantly reoriented relative to the compound-nucleus spin).

The center-of-mass $\alpha$-particle spectra observed near $\theta_{\text {c.m. }}=90^{\circ}$ for four $k_{\gamma}$ gates are shown in Fig. 2. The spectral-shapes do not change significantly with fold and are well described by one evaporative component with a peak near $15 \mathrm{MeV}$. However, as a function of angle with respect to the derived spin direction, there are differences which can best be seen in angular distributions of $\alpha$ particles of given energy with respect to the spin direction. This is shown in Fig. 3 where these correlations are presented for $\alpha$-particle energies of 13 and 20 $\mathrm{MeV}$ for a low- $k_{\gamma}$ gate $(9-13)$ and for $13 \mathrm{MeV}$ for $k_{\gamma}$ gates of 14-18 and 19-23. For constant $\alpha$-particle ener- 


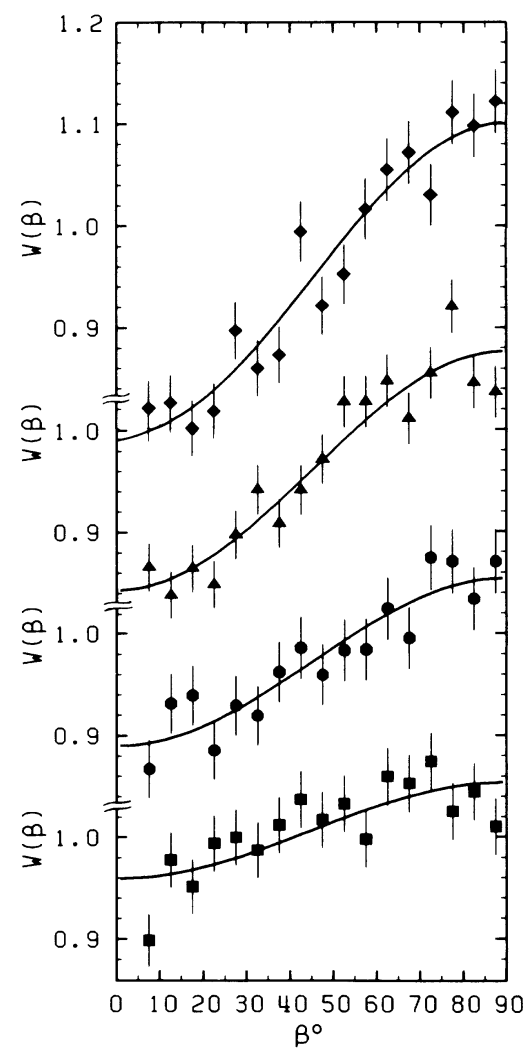

FIG. 3. Examples of experimental angular distributions of $\alpha$ particles with respect to the spin direction of the residual nucleus. The squares and circles correspond to $k_{r}=9-13$ for $E_{a}=13$ and $20 \mathrm{MeV}$, respectively; the triangles and diamonds correspond to $E_{a}=13 \mathrm{MeV}$ for the $k_{r}$ gates 14-18 and 19-23, respectively. The solid lines are least-squares fits of $A_{0}$ $\times\left[1+A_{2} P_{2}(\cos \beta)\right]$ to the data.

gy $(13 \mathrm{MeV})$, as $k_{\gamma}$ increases the anisotropy of the correlation increases in favor of $\alpha$ emission perpendicular to the spin direction. It is also seen that, for a given $k_{\gamma}$ gate, the anisotropy increases in the direction of emission perpendicular to spin as the $\alpha$-particle energy increases.

In order to show the energy dependence of the observed anisotropies from the ${ }^{110} \mathrm{Sn}$ compound system, the measured angular distributions were fitted as a function of $E_{\alpha}$ to $W(\beta)=A_{0}\left[1+A_{2} P_{2}(\cos \beta)\right]$. These fits are shown in Fig. 3 as the solid lines. The $A_{2}$ coefficients are displayed in Fig. 4(a) for three $k_{\gamma}$ bins. It is clearly seen that for all $k_{\gamma}$ bins the $A_{2}$ coefficients monotonically increase (anisotropies decrease) with decreasing $E_{\alpha}$ for values well above to values well below the exit-channel Coulomb barrier. It is also seen that the anisotropies increase with increasing fold. The anisotropies for a fourth bin, $k_{\gamma}=24-30$, were comparable to those for the $k_{\gamma}=19-23 \mathrm{bin}$. The four $k_{\gamma}$ gates were converted to $M_{\gamma}$ and then to $I_{\mathrm{ER}}$ as described by Sarantites et al. ${ }^{7}$ The above $k_{\gamma}$ bins correspond to $M_{\gamma}$ bins of 8-15, 13-20,
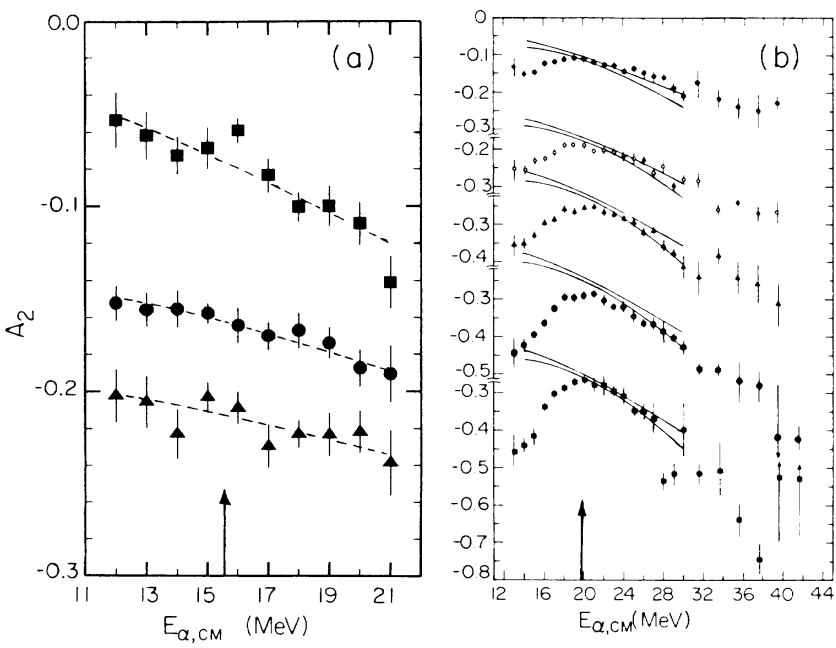

FIG. 4. (a) $A_{2}$ coefficients as functions of $E_{a}$ from ${ }^{110} \mathrm{Sn}$ decay. The points represent the experimental results. The squares, circles, and triangles correspond to $k_{r}$ bins of 9-13, 14-18, and 19-23, respectively. The dashed lines are drawn to guide the eye. (b) $A_{2}$ coefficients from ${ }^{170} \mathrm{Yb}$ decay, from Ref. 4. The closed and open circles correspond to $k_{\gamma}$ bins of 11-14 and 15-18 ( $I \sim 34$ and 43$)$, respectively. The closed triangles, closed large circles, and closed squares correspond to $k_{y}$ bins of $19-22,23-26$, and $27-32(I-51,59$, and 64), respectively. The pairs of curves are full-width half-maximum boundaries of the spherical Monte Carlo calculation of the $A_{2}$ coefficients. The arrows in (a) and (b) indicate the position of the spherical barrier calculated with $r_{0}=1.4 \mathrm{fm}$.

17-23, and 20-25. Statistical-model calculations using the code JULIAN-PACE of Hillman and Eyal ${ }^{8}$ were used to estimate the corresponding average spins of the evaporation residues (ER's) prior to $\gamma$ decay and of the $\alpha$ emitting nuclei. The average spin values for the ER were found to be $20 \pm 4,25 \pm 4,29 \pm 3$, and $33 \pm 2$, while those for the $\alpha$-emitting nuclei were $36 \pm 10$, $41 \pm 8,43 \pm 7$, and $46 \pm 7$, respectively. The average $Z$ and $A$ of the $\alpha$-emitting nuclei were also estimated from $\mathrm{PACE}^{8}$ to be $48 \pm 2$ and $107 \pm 2$, respectively.

For comparison the $A_{2}$ coefficients for the ${ }^{170} \mathrm{Yb}$ system are shown in Fig. 4(b). The different trends in the experimental correlations for the two systems are clearly seen for $E_{a}$ below the spherical Coulomb barriers which are marked by the vertical arrows. The decrease in $A_{2}$ for $E_{\alpha}$ below the barrier is not observed in the Sn case contrary to observation for the $\mathrm{Yb}$ system [Fig. 4(b)]. The solid lines in Fig. 4(b) show the $A_{2}$ coefficients for the $\mathrm{Yb}$ system predicted ${ }^{4}$ by the statistical model which uses transmission coefficients for a spherical system. These calculations show a monotonic decrease in anisotropy (increase in $A_{2}$ ) as the energy decreases well below the barrier in contrast with observation for the $\mathrm{Yb}$ system but in agreement with the findings for the Sn system. 
In our earlier work, ${ }^{4}$ we argued that the increase in the $\alpha$ anisotropy below the barrier is qualitatively the behavior expected for a deformed $\alpha$-emitting system. The behavior which we observe for the ${ }^{110} \mathrm{Sn}$ compound system is just that predicted ${ }^{4}$ for a spherical system. The inferences concerning the shape of $\mathrm{Yb}$ and $\mathrm{Sn}$ compound systems at high spin and excitation energy are strongly supported by observation of the decay of giant dipole resonances built on excited states. The data for ${ }^{166} \mathrm{Er}$ (formed by $84-\mathrm{MeV}{ }^{16} \mathrm{O}$ on ${ }^{150} \mathrm{Nd}, E^{*}=61.5 \mathrm{MeV}$ ) exhibit a broadened peak suggesting a two-component resonance in distinct contrast to observations for a ${ }^{108} \mathrm{Sn}$ compound system (formed by $84-\mathrm{MeV}{ }^{16} \mathrm{O}+{ }^{92} \mathrm{Mo}$, $E^{*}=61.2 \mathrm{MeV}$ ) where only a single resonance peak was observed. $^{5}$ These systems ${ }^{166} \mathrm{Er}$ and ${ }^{108} \mathrm{Sn}$ are indeed very similar to those studied here. Furthermore, recent calculations ${ }^{9}$ based on a cranked deformed Nilsson potential predict that for temperatures of $<1.6 \mathrm{MeV}$ and $J<40{ }^{108} \mathrm{Sn}$ remains spherical or slightly oblate.

In summary, we have measured angular distributions of $\alpha$ particles with respect to the spin direction for two hot, rapidly rotating compound systems, ${ }^{170} \mathrm{Yb}$ and ${ }^{110} \mathrm{Sn}$. The absence of an increasing anisotropy for subbarrier $\alpha$ particles from the ${ }^{110} \mathrm{Sn}$ system in contrast to ${ }^{170} \mathrm{Yb}$ indicates that $\alpha$ emission from $\mathrm{Sn}$ is consistent with emission from a spherical system. For the ${ }^{170} \mathrm{Yb}$ system deformation is needed to explain the data. We suggest that angular distributions of subbarrier alphas provide a sensitive method for probing nuclear deformations of hot, highly spinning nuclei.

This work was supported in part by the U. S. Depart- ment of Energy under Contract Nos. DE-AS0276ER04052 and DE-AC02-85ER40210. One of us (L.G.S.) acknowledges support from a U. S. Presidential Young Investigator Award. Oak Ridge National Laboratory is operated by Martin Marietta Energy Systems Inc. under Contract No. DE-AC05-84ER212400 with the U. S. Department of Energy. Two of us (R.P.S. and G.N.) acknowledge support from the Robert A. Welch Foundation.

\footnotetext{
(a) Permanent address: Institute of Physics, Jagellonian University, Kraków, Poland.

(b) Present address: Department of Physics, University of Jyväskylä, Jyväskylä, Finland.

(c) Present address: Hughes Aircraft Co., EDSG, P. O. Box 902, MS E0/E2/S107, El Segundo, California 90245.

${ }^{1}$ J. O. Newton et al., Phys. Rev. Lett. 46, 1383 (1981).

${ }^{2}$ G. F. Bertsch and R. A. Broglia, Phys. Today 39, 44 (1986).

${ }^{3}$ M. Jääskeläinen et al., Nucl. Instrum. and Methods Phys. Res. 204, 385 (1983).

${ }^{4}$ F. A. Dilmanian et al., Phys. Rev. Lett. 49, 1909 (1982).

5J. J. Gaardhøje et al., Phys. Rev. Lett. 53, 148 (1984).

${ }^{6}$ D. G. Sarantites et al., in High Angular Momentum Properties of Nuclei, edited by N. R. Johnson (Harwood, New York, 1983), p. 417.

${ }^{7}$ D. G. Sarantites et al., Phys. Lett. 115B, 441 (1982).

${ }^{8} \mathrm{M}$. Hillman and Y. Eyal, code JULIAN, unpublished; A. Gavron, modification PACE, Phys. Rev. C 21, 230 (1980).

${ }^{9}$ M. Gallardo et al., Nucl. Phys. A443, 415 (1985).
} 